\title{
Acknowledgment to Reviewers of Chemosensors in 2020
}

\section{Chemosensors Editorial Office}

MDPI AG, St. Alban-Anlage 66, 4052 Basel, Switzerland

Peer review is the driving force of journal development, and reviewers are gatekeepers who ensure that Chemosensors maintains its standards for the high quality of its published papers. Thanks to the cooperation of our reviewers, in 2020, the median time to first decision was 16 days and the median time to publication was 38 days. The editors would like to express their sincere gratitude to the following reviewers for their precious time and dedication, regardless of whether the papers were finally published:

Aastrup, Teodor

Abdi, Frank

Abdulhalim, Ibrahim

Adachi, Naoya

Adhikari, Bal Ram

Afify, Ahmed

Alam, Arif Ul

Aldewachi, Hasan

Aleixandre, Manuel

Altintas, Zeynep

Ananias, Duarte

Antunes, Joana C.

Ashley, Jon

Ashrafi, Amirmansoor

Ata, Seisuke

Baldini, Francesco

Bane, Susan L.

Barek, Jiri

Barek, Jiří

Barille, Regis

Barrientos Velazquez, Ana Luisa

Barros, Joana

Baù, Marco

Bayer, Ilker

Bearzotti, Andrea

Bec, Krzysztof

BelBruno, Joseph

Bento, Fatima

Bessmertnykh-Lemeune, Alla

Bollella, Paolo

Boytsova, Olga V.

Bratov, Andrey

Bukauskas, Virginijus

Cabaj, Joanna

Cai, Jinguang
Calvino, Céline

Campanella, Luigi

Canet Ferrer, Josep

Cannatà, Domenico

Carruthers, David

Chang, Chia-Chen

Chang, Suk-Kyu

Chen, Chien-Fu

Chen, Hui

Chen, Min-Hua

Chernysheva, Maria G.

Chiavaioli, Francesco

Chinh, Nguyen Duc

Cho, Byungjin

Cho, Eun Jeong

Choi, Bong-Gil

Choi, Kyung Hyun

Choi, Seungkeun

Chung, Yoonyoung

Ciasca, Gabriele

Clement, Pierrick

Compagnone, Dario

Correa, Daniel S.

Correa, Diego Rafael Nespeque

Cummins, Gerard

Da Silveira Petruci, João Flávio

De Almeida, Jose Manuel

De Araujo, William Reis

De Lannoy, Charles-François

De Oliveira, Helinando Pequeno

De Salas, Felipe

De Stefano, Luca

Denisov, Ivan A.

Dhanjai, Dhanjai

Dilonardo, Elena 
Dossi, Carlo

$\mathrm{Du}$, Xin

Duhamel, Jean

Duma, Luminita

Dunbar, Alan

El-Aassar, Mohamed

Elena, Husanu

Eleni, Makarona

El-Moghazy, Ahmed

El-Sayed Okeil, Sherif

Evtugyn, Gennady

Felix, Anderson André

Fiedler, Holger

Finlayson, Chris

Forzani, Erica

Frense, Dieter

$\mathrm{Fu}$, Qiang

Gabriele, Magna

Gan, Shiyu

Gancarz, Marek

Gao, Zhuangqiang

Gębicki, Jacek

Ghibaudo, Gérard

Ghosal, Sutapa

Ghosh, Kalyan

Giungato, Pasquale

Głuchowski, Paweł

Gnyba, Marcin

González-Vera, Juan A.

Gorodkiewicz, Ewa

Grimaldi, Maria Grazia

Guglielmino, Salvatore

Gullett, Brian K.

Hamedpour, Vahid

Han, Guo-Cheng

Han, Shuhua

Happy, Henri

Hashishin, Takeshi

He, Qiyuan

Heift, Dominikus

Hernández-Núñez, Emanuel

Hess-Dunning, Allison

Hinman, Sam

Hong, Jun

Hortigüela, María J.

Horvai, George

Hosu, Oana

Hsu, Huan-Hsuan

$\mathrm{Hu}$, Yonggang

Huang, Qijin

Huang, Wen Chang
Huang, Xianhe

Il, Jeon

Imamura, Gaku

Iqbal, Hafiz M. N.

Isoaho, Noora

Jaffrezic-Renault, Nicole

Jakieła, Sławomir

James, Nicholas G.

Janegitz, Bruno Campos

Jia, Yunfang

Jmerik, Valentin

Johnson, Scooter

Juang, Yi-Je

Jurczak, Janusz

Kadam, Avinash

Kahn, Myrtil L.

Kammer, Julien

Kandadai, Nirmala

Kaneti, Yusuf Valentino

Kang, ChanKyu

Karthik, Raj

Kawakami, Jun

Khalid, Muhammad

Kim, Dong-Joo

Kim, Young-Pil

Kirchner, Eric

Kitazawa, Takafumi

Kolmakov, Andrei

Koren, Klaus

Koudelková, Zuzana

Lan, Minbo

Le Borgne, Brice

Lee, Tae Yoon

Lee, Woo Sung

Levine, Mindy

Liang, Zhifang

Lima, Jose Luiz Sousa

Lin, Tzu-En

Lin, Yang-wei

Liu, Chung-Chiun

Liu, Weilu

Liu, Yu

Llopis-Lorente, Antoni

Lo Faro, Maria Josè

Lopes, Paula

Lova, Paola

Lowry, Mark

Lu, Dingqiang

Lu, Jin

Lubal, Přemysl

Lvova, Larisa 
Maćkowski, Sebastian

Macwan, Isaac

Magna, Gabriele

Maier, Guenther A.

Majerus, Steve

Majewska, Katarzyna

Malla, Spundana

Mandal, Krishna C.

Mani, Ganesh Kumar

Maniam, Subashani

Martins, Verónica C.

Mascini, Macello

Massera, Ettore

Masud, Jahangir

Matsui, Jun

Mauro, Nicolò

Medvidović-Kosanović, Martina

Meng, Fanli

Meng, Hong

Mi, Xianqiang

Miglietta, Maria Lucia

Mikołajczyk, Tomasz

Miricioiu, Marius

Montes García, Verónica

Mourzina, Yulia

Muguruma, Hitoshi

Müller, Gerhard

Munoz-Aguirre, Severino

Muranaka, Atsuya

Murtada, Khaled

Murugappan, Krishnan

Myasoedova, Tatiana N.

$\mathrm{Na}$, Jun-Hee

Nagles, Edgar

Navratil, Tomas

Ndieyira, Joseph

Nima, Zeid A.

Nimse, Satish Balasaheb

Novotny, Vit

O'Hare, Danny

Oliveira Salles, Maiara

Olson, Mark

Olthuis, Wouter

Othman, Ali

Otř́sal, Pavel

Palanisamy, Selvakumar

Palys, Barbara

Palzer, Stefan

Pandey, Sadanand

Pandíţh, Anúp

Panzardi, Enza
Park, Ki Soo

Park, Min

Paterno, Leonardo Giordano

Pauliukaite, Rasa

Pedras, Bruno

Peng, Zhili

Peres, Henrique E. M.

Persaud, Krishna

Petousis, Markos

Pilan, Luisa

Pingarrón, José

Pinto Da Silva, Luís

Piszczek, Piotr

Plusquellic, David F.

Pozdin, Vladimir

Puchades, Ivan

Puton, Jarosław

Qiu, Xianbo

Quevedo, Ivan R.

Radadia, Adarsh D.

Radwan-Pragłowska, Julia

Ramanavicius, Arunas

Ramos-Ortiz, Gabriel

Rana, Abu Ul Hassan Sarwar

Raymundo-Pereira, Paulo A.

Reiss, Lucinda V.

Richards, Chris

Riek, Roland

Rivera, Ernesto

Rodenbücher, Christian

Rodríguez, José Antonio

Rusinek, Robert

Rutyna, Iwona

Rydosz, Artur

Sakač, Nikola

Salcedo, Walter J.

Saruhan, Bilge

Satrapinskyy, Leonid

Sberveglieri, Veronica

Scherf, Ullrich

Schneider, Rudolf

Schroeder, Grzegorz

Schwans, Jason P.

Sedláček, Petr

Semancik, Stephen

Sengottuvelu, Dineshkumar

Serrano, Núria

Sgobbi, Lívia Florio

Shafagh, Reza Zandi

Shan, Xin

Shan, Xuechuan 
Sierra Rosales, Paulina

Silva, Gabriela Ventura

Singh, Parvesh

Smetana, Michael

Spyridon, Blionas

Squella, Juan Arturo

Stobiecka, Magdalena

Sun, Jing Zhi

Sun, Jinhua

Suzurikawa, Jun

Szafranski, Szymon

Szkoda, Mariusz

Szuber, Jacek

Tai, Huiling

Tangirala, Venkata Krishna Karthik

Teixeira, Marcos F. S.

Tong, Yexiang

Trammell, Scott A.

Tripathi, Kumud Malika

Tsagkaris, Aristeidis

Tsai, Hsieh-Fu

Tsay, Chien-Yie

Tsizh, Bohdan

Tu, Yifeng

Usama, Syed Muhammad

Valero, Edelmira

Vargas-Rodriguez, Everardo

Velotta, Raffaele

Vestergaard, Mun'delanji C.

Vidic, Jasmina

Villarreal-Gómez, Luis Jesús

Vincenzo, De Leo

Vitale, Flavia

Volmajer, Julija

Vozzi, Giovanni

Vrcek, Ivana Vinkovic

Wang, Hancong

Wang, Huxuan

Wang, Luyu

Wang, Qingxiang

Wang, Xiaofeng
Wang, Yanyan

Wang, Zhenxin

Ward, Andrew

Wei, Qingshan

Weng, Wei

Wilson, Dan

Wolski, Karol

Wong, Lai Mun

Wu, Chunsheng

$\mathrm{Wu}$, Han-Chun

$\mathrm{Wu}$, Jian

Wyszyński, Dominik

Xavier, Jolly

Xian, Xiaojun

Xiao, Xinxin

Xie, Kefeng

Xu, Guobao

Yamaguchi, Yuki

Yang, Yi

Yoshino, Toshihiko

Yu, Fabiao

Yu, Hakki

Zaidi, Shabi Abbas

Zamfir, Lucian-Gabriel

Zangeneh-Nejad, Farzad

Zarejousheghani, Mashaalah

Zeng, Wen

Zhang, Haoran

Zhang, Jintao

Zhang, Mingzhe

Zhang, Ruixiong

Zhang, Shaolin

Zhao, Huimin

Zhao, Jiang

Zhao, Zhan

Zhou, Shan

Zhu, Liping

Zhu, Zijie

Zuffo, Michela

Zwergel, Clemens 